\title{
COVID-19 herd immunity v. learning to live with the virus
}

\author{
S A Madhi, MB BCh, PhD \\ South African Medical Research Council Vaccines and Infectious Diseases Analytical Research Unit, Faculty of Health Sciences, University of \\ the Witwatersrand, Johannesburg, South Africa; and African Leadership in Vaccinology Expertise, Faculty of Health Sciences, University of the \\ Witwatersrand, Johannesburg, South Africa
}

Corresponding author: S A Madhi (shabir.madhi@wits.ac.za)

\begin{abstract}
Mutations of SARS-CoV-2 have been associated with increased transmissibility and occasionally reduced sensitivity to neutralising antibody activity induced by past ancestry virus infection or current COVID-19 vaccines. Nevertheless, COVID-19 vaccines have consistently demonstrated high efficacy and effectiveness against COVID-19 severe disease, hospitalisation and death, including disease caused by designated variants of concern. In contrast, COVID-19 vaccines are more heterogeneous in reducing the risk of infection and mild COVID19, and are modestly effective in interrupting virus transmission. Ongoing mutations of SARS-CoV-2 resulting in increased transmissibility and relative evasion of neutralising antibody activity induced by past virus infection or COVID-19 vaccines are likely. The duration of protection induced by COVID-19 vaccines is modelled to be relatively short in protecting against infection and mild COVID-19, but is likely to be 2 - 3 years against severe disease. Current experience from the UK and Israel demonstrates that even with high levels of COVID19 vaccine coverage ( $>85 \%$ of the adult population), resurgences with new variants of concern remain a strong probability. Nevertheless, such resurgences are not mirrored by high rates of hospitalisation and death compared with what was experienced in relatively COVID-19 vaccine-naive populations. Even though COVID-19 vaccines are unlikely to result in a herd immunity state, their ability to protect against severe COVID-19 and death could allow for a return to normalcy once a large enough proportion of the adult population in a country has been vaccinated.
\end{abstract}

S Afr Med J 2021;111(9):852-856. https://doi.org/10.7196/SAMJ.2021.v111i9.16005

\section{The burden of COVID-19}

It has been 18 months since the World Health Organization declared SARS-CoV-2 disease (COVID-19) a pandemic, with 197.4 million cases and 4.22 million COVID-19 deaths reported by 1 August 2021. ${ }^{[1]}$ The number of documented COVID-19 cases and deaths, however, probably grossly under-estimates the burden of COVID-19, particularly in low- and middle-income countries (LMICs) where access to SARS-CoV-2 testing remains constrained. South Africa (SA) has the highest testing rate for SARS-CoV-2 in Africa (cumulative 248 per 1000 population), which is $1 / 14$ th of the cumulative testing rate in the UK ( 3516 per 1000 population). ${ }^{[1]}$ Corroborating that the burden of SARS-CoV-2 infection has been under-estimated in SA is the sero-survey (testing for antibody to determine past infection) which reported that $32-63 \%$ of blood donors across four provinces in SA had been infected by SARS-CoV-2 prior to the current resurgence (third wave). ${ }^{[2]}$ Consequently, the 2.39 million recorded COVID-19 cases ( $4 \%$ of the population) probably represents $1 / 10$ th of the SARS-CoV-2 infections that have occurred in SA. The immense force of SARS$\mathrm{CoV}-2$ infection experienced in SA transpired despite attempts by the government to limit the rate of its spread through highly restrictive restrictions, which massively impacted on the economy and livelihoods of people.

Similarly, the burden of reported COVID-19 deaths is probably an under-estimate globally, particularly in LMICs with fragile healthcare systems and tenuous vital registration registries unable to track trends in mortality in real time. In India alone, modelling of excess deaths attributable to COVID-19 (i.e. deaths over and above what would be expected based on historical averages) using different data sources and case scenarios estimates that there had been $3.4-4.9$ million COVID-19 deaths (crude estimated death rate 261 - 360 per 100000 population) by July 2021, which is approximately 10 -fold higher than the 423000 documented deaths (crude estimated death rate 31 per
100000 ) by July $2021 .^{[3]}$ In SA, the modelled excess mortality since May 2020 has been strikingly synchronous with documented COVID19 deaths, indicating that the vast majority of the excess deaths were due to COVID-19. This is corroborated by data from the Western Cape, the province with the most robust public health surveillance and healthcare system in the country, where the recorded COVID-19 and modelled excess deaths are closely aligned. In contrast, there is a huge discrepancy between recorded and modelled excess deaths in Gauteng, where $25 \%$ of the SA population lives. ${ }^{[4]}$ The death toll of COVID-19 in SA as of July 2021 is likely to be closer to 214200 (crude mortality rate 360 per 100000 population aged $>1$ year) rather than the underestimated 70388 (118 per 100000$)$ recorded deaths.

Given this background, it is important to reflect on the success against and challenges posed by COVID-19, to chart a sustainable pathway forward with the goal of minimising further direct and indirect suffering due to COVID-19 in SA and globally.

\section{Evolution of SARS-CoV-2 and implications for herd immunity}

In the absence of ability to contain the spread of SARS-CoV-2 in the vast majority of countries, herd immunity was widely envisioned and propagated as the endgame for COVID-19. A minority of scientists and politicians advocated for minimal interventions and to allow SARS-CoV-2 infections to run their course, in the misguided belief that it would result in a herd immunity threshold being reached. Such a strategy was advocated oblivious to the cost of human life that would have transpired. Also, whether herd immunity would result through natural infection alone was doubtful. With the ongoing evolution of the virus, including mutations that made it more infectious, more transmissible and relatively resistant to immunity induced by past infection or by first-generation COVID-19 vaccines, herd immunity is now an aspiration unlikely to materialise. 
The dynamic mutations of SARS-CoV-2 over the past 6 - 8 months, coupled with the now inequitable and sluggish roll-out of COVID19 vaccines, call into question whether herd immunity can be attained even with acceleration of COVID-19 vaccine coverage. Reaching a herd immunity threshold is more than balancing a simple mathematical equation $\left(p_{1}=1-1 / R_{0}\right)$. In this equation, $p_{1}$ is the proportion of immune individuals, i.e. who are no longer at risk of being infected by the micro-organism (or at least able to clear it very quickly without onward transmission to others). The reproductive rate $\left(R_{0}\right)$ is the average number of individuals who will be infected by the micro-organism in a fully susceptible population by a new case. Over time, as there is a build-up of people who develop immunity against infection by the virus (which is very different to being protected against COVID-19 or severe disease), the effective reproductive rate gravitates to $<1$ (i.e. every new case will on average infect less than one other person). The requirement for the effective reproductive rate being sustained at $<1$ in the absence of other interventions that might affect the rate of transmission of the micro-organism, such as social distancing, wearing of face masks and avoiding mass gatherings, is when herd immunity can be declared.

Challenges posed by COVID-19 include the fact that the transmissibility and infectiousness of SARS-CoV-2 have increased over time owing to evolving mutations of the spike protein. The $R_{0}$ for the Wuhan strain of SARS-CoV-2 was estimated to be $2.5-4.0{ }^{[5]}$ The $R_{0}$ is dynamic and influenced by local factors such population density, population structure and main sources of transmission, living environment conditions and patterns of social interaction. Also, implementation of restrictions on social activities, adoption of masking and other non-pharmacological interventions would affect the virus transmission rate. SARS-CoV-2 mutations that resulted in variants of concern such as the delta variant now dominate almost globally, and are estimated to be two-fold more transmissible than the ancestry virus. ${ }^{[6]}$ Hence, whereas the simple mathematical equation predicted that herd immunity could be reached once $67 \%(67 \%=$ $1-(1 \div 3) \times 100 \%$, assuming $\left.R_{0}=3\right)$ of the population developed adequate immunity against infection by the ancestry virus, the threshold would increase to $84 \%$ (assuming $R_{0}=6$ ) for the delta variant.

\section{SARS-CoV-2 and immune evasion}

The need to recalibrate our expectations of whether herd immunity can be attained, the role of COVID-19 vaccines and the future of the COVID-19 pandemic was prominently highlighted following the identification of the beta variant in SA in November 2020. The evolution of the beta and other variants of concern (such as alpha, gamma and delta) emerged partly as a result of build-up of immune pressure through natural acquired SARS-CoV-2 infections and prior to the widespread use of COVID-19 vaccines. An increased prevalence of antibody against SARS-CoV-2 induced by ongoing virus transmission and sluggish roll-out of COVID-19 vaccine in most parts of the world is likely to facilitate further mutations as the virus continues to adapt.

The relative resistance of the beta variant to in vitro neutralising activity of antibody induced by ancestry SARS-CoV-2 infection ${ }^{[7]}$ provided the first signal of challenges faced in the agenda to achieve a herd immunity state. A longitudinal cohort study in SA demonstrated that past infection by the ancestry virus did not provide protection against SARS-CoV-2 infection by the beta variant. ${ }^{[8]}$ This was compounded by findings of the University of Oxford/AstraZeneca non-replicating simian adenovirus spike-protein vaccine (AZD1222) in SA, which despite demonstrating $75 \%$ protection against mild to moderate COVID-19 caused by the ancestry virus 14 days after a single dose, showed no efficacy against mild to moderate beta-variant COVID-19 following two doses of the vaccine. ${ }^{[9]}$ The reduction in vaccine-induced neutralising antibody activity against the beta variant has been demonstrated for all COVID-19 vaccines, albeit varying between vaccines. ${ }^{[10,11]}$

A definitive correlate of protection against SARS-CoV-2 infection is yet to be established. Nevertheless, the magnitude of vaccineinduced fold increase in in vitro neutralising (correlation coefficient $=$ 0.79 ) and binding antibodies (correlation coefficient $=0.93$ ) is strongly associated with observed efficacy against COVID-19 study endpoints enriched for mild to moderate COVID-19. ${ }^{[12]}$ Notably, COVID-19 vaccine-induced neutralising antibody activity is variably affected by different variants of concern. In SA, the beta variant that dominated during the second COVID-19 wave was shown to have near to complete resistance to antibody-neutralising activity induced by past infection by the ancestry virus and the AstraZeneca COVID-19 vaccine, ${ }^{[4,5]}$ as well as greatly diminished neutralising activity induced by messenger RNA (Pfizer-Biontech and Moderna) COVID-19 vaccines compared with the neutralising activity against the ancestry virus. ${ }^{[6]}$

The differences in magnitude of COVID-19-induced neutralising antibody against the beta variant are likely to contribute to varying effectiveness against mild to moderate COVID-19, with the PfizerBiontech vaccine (BNT162b2), which induces a high magnitude of neutralising antibody against the ancestry virus, able to overcome some of the resistance of the beta variant and consequently reporting $100 \%$ efficacy against the beta variant. ${ }^{[13]}$

Although the currently dominating delta variant is more susceptible to vaccine-induced neutralising antibody activity compared with the beta variant, it too shows intermediary resistance to vaccine-induced neutralising antibody compared with the ancestry virus. ${ }^{[10,14]}$

Whereas variant-specific neutralising antibody activity appears to be associated with efficacy against mainly mild to moderate COVID$19,{ }^{[12,15]}$ an immunological biomarker associated with protection against severe COVID-19 remains to be ascertained. Despite near abrogation of AZD1222-induced in vitro neutralising antibody activity following two doses of the vaccine, vaccination with the AZD1222 vaccine in a Syrian hamster SARS-CoV-2 challenge model protected against any lung pathology following beta-variant challenge, despite not preventing viral replication in the nose. ${ }^{[16]}$ Furthermore, vaccine effectiveness for the AstraZeneca vaccine against COVID-19 hospitalisation or death due to the beta or gamma variants, both of which share the E484K mutation, was $83 \%$ (95\% confidence interval 66 - 92) . $^{[17]}$ These data indicate that the magnitude of neutralising and binding antibody is strongly correlated to protection against SARS-CoV-2 infection and mild to moderate disease. In contrast, either much lower concentrations of neutralising antibody may be required to protect against severe COVID-19, or - more compelling - protection against severe COVID-19 is possibly mediated by T-cell immunity. The CD4+ and CD8+ (natural killer cell) responses induced by the AZD1222 vaccine were generally unaffected by the spike protein mutations in the beta variant. ${ }^{[9]}$

\section{Examples of impact of COVID-19 immunisation}

Israel and the UK are countries with among the highest COVID-19 vaccine coverage rates globally, at $61.6 \%$ and $55.2 \%$, respectively, including $\sim 85 \%$ of the adult population having been vaccinated. ${ }^{[1]}$ The experience of the COVID-19 immunisation programme in these two countries, however, provides further evidence of the unlikelihood that herd immunity would materialise against COVID- 
19. Israel has exclusively used the BNT162b2 vaccine, which together with the Moderna and Novavax vaccines, induces the greatest fold increase in neutralising antibody responses after vaccination relative to antibody in convalescent sera (i.e. antibody in people recovering from COVID-19). ${ }^{[12.15]}$

Despite persistence of T-cell responses and IgG memory B-cells being evident at least $9-12$ months after infection (and possibly also following vaccination), ${ }^{[18]}$ significant declines in neutralising antibody are observed as early as 76 days after two doses of the BNT162b2 and AZD1222 vaccines. ${ }^{[19]}$ Waning of neutralising antibody activity, coupled with a shift from the alpha to the more transmissible delta variant, could explain the decrease in effectiveness of the BNT162b2 vaccine in Israel. Soon after the introduction of the BNT162b2 vaccine in Israel, when the alpha variant mainly dominated, direct and indirect effects of vaccination were observed. In addition to BNT162b2 reducing the risk of hospitalisation by $94 \%$ in Israel, vaccination also protected against any symptomatic COVID-19 (92 - 97\% risk reduction) and asymptomatic illness ( $84 \%$ risk reduction), ${ }^{[20-22]}$ and reduced infectiousness by $41-78 \%,{ }^{[23,24]}$ possibly owing to vaccinated individuals having lower viral loads. ${ }^{[25]}$ These data boded well that rapid scale-up and high coverage with COVID-19 vaccines could lead to herd immunity.

Unfortunately, the emergence of the delta variant in Israel was associated with a resurgence of COVID-19, mainly presenting as mild illness. ${ }^{[1]}$ Early estimates from Israel report that vaccine effectiveness against COVID-19 of any severity has waned from the initial $92-94 \%$ to $39 \%$, albeit retaining $91 \%$ effectiveness against severe illness. ${ }^{[2]}$ Factors contributing to the COVID-19 resurgence in Israel may include: (i) waning of vaccine-induced antibody over time; (ii) the delta variant being $40-60 \%$ more transmissible than the alpha variant that dominated early on in the vaccination programme, and possibly requiring a high concentration of neutralising antibody to protect against infection; and (iii) the delta variant being twofold less sensitive to vaccine-induced neutralising antibody relative to the ancestry virus or alpha variant. Also, these findings suggest that although IgG-producing memory B-cells may persist for at least 9 - 12 months after natural infection, ${ }^{[18]}$ mobilisation of these memory B-cells may not be timeous enough to protect against infection, even by variants relatively susceptible to vaccine-induced neutralising antibody activity. The Israeli experience attests to the challenges likely to be experienced in reaching and sustaining a herd immunity state, more so as SARS-CoV-2 is likely to undergo ongoing mutations that will be near to impossible to restrict to any geographical locality.

Similarly, in the UK, where the AZD1222 and BNT162b2 vaccines are mainly being deployed, despite high levels of vaccine coverage there has been a resurgence of COVID-19, also predominantly due to the delta variant (Fig. 1). The current COVID-19 wave in the UK is subsiding, having peaked at a lower level than experienced during the second wave, which occurred at an early stage of roll-out of the COVID-19 vaccine and was caused by the alpha variant, which is relatively less infectious than the delta variant. ${ }^{[1]}$ It is possible that despite delta-variant breakthrough infections occurring after vaccination, immunity induced by the vaccines results in a lower viral load and duration of shedding. Such an effect of vaccines could assist in reducing virus transmission and attenuating the magnitude of future resurgences, even if it is not sufficient to achieve a herd immunity state.

Another feature of the current resurgences in the highly vaccinated Israeli and UK populations is the unhinging in trends of COVID-19 cases and deaths compared with the earlier waves (Figs 2 and 3). The excess mortality in the UK was $>100 \%$ and $40 \%$ of that expected at the time of the peak of the first and second waves, respectively. In contrast, excess deaths being experienced with the current outbreak are already subsiding in the UK and were only $5 \%$ above the historical average for the corresponding period (Fig. 2). Similarly, in Israel, where excess deaths attributable to COVID-19 were $40 \%$ above what was expected at the peak of the outbreak prior to COVID-19 vaccine roll-out, the current estimate for excess deaths in a population with high vaccine coverage is $\sim 10 \%$ (Fig. 2 ).

The experience from both the UK and Israel emphasises that COVID-19 vaccines have greatest value in reducing the risk of severe

The number of confirmed cases is lower than the number of actual cases, mainly because of limited testing.

Note: Head-to-head comparisons across the wave and between countries are misleading owing to differences in testing

rates and criteria used for testing of suspected cases or infections.

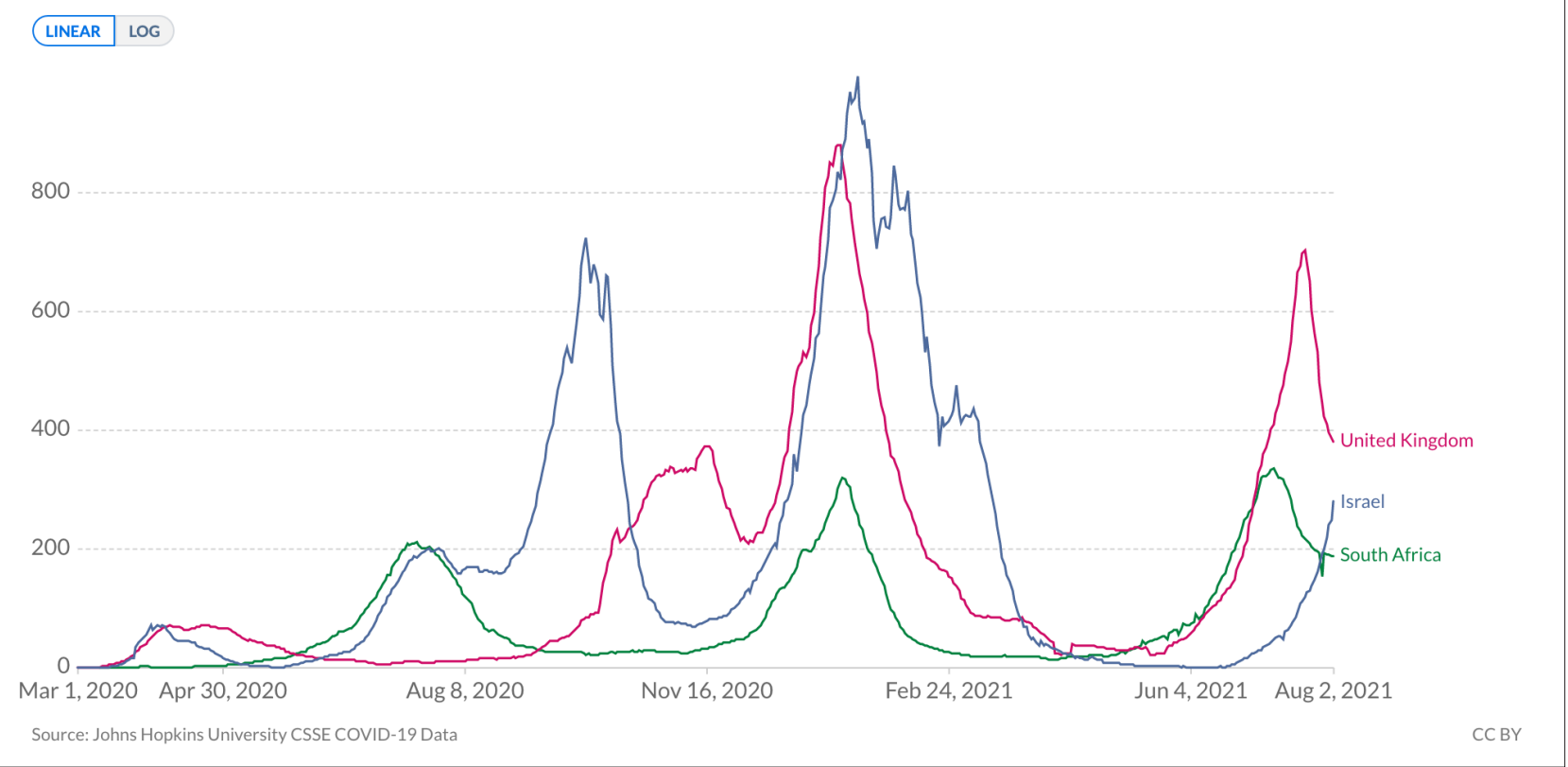

Fig. 1. Daily COVID-19 cases per million population in Israel, the UK and South Africa (source: Our World in Data ${ }^{[1]}$ ). The rolling 7-day average is shown. 
Shown is how the number of weekly or monthly deaths in 2020 - 2021 differ as a percentage from the average number of deaths in the same period over the years 2015 - 2019. This metric is called the P-score. The reported number of deaths might not count all deaths that occurred, owing to incomplete coverage and delays in death reporting.

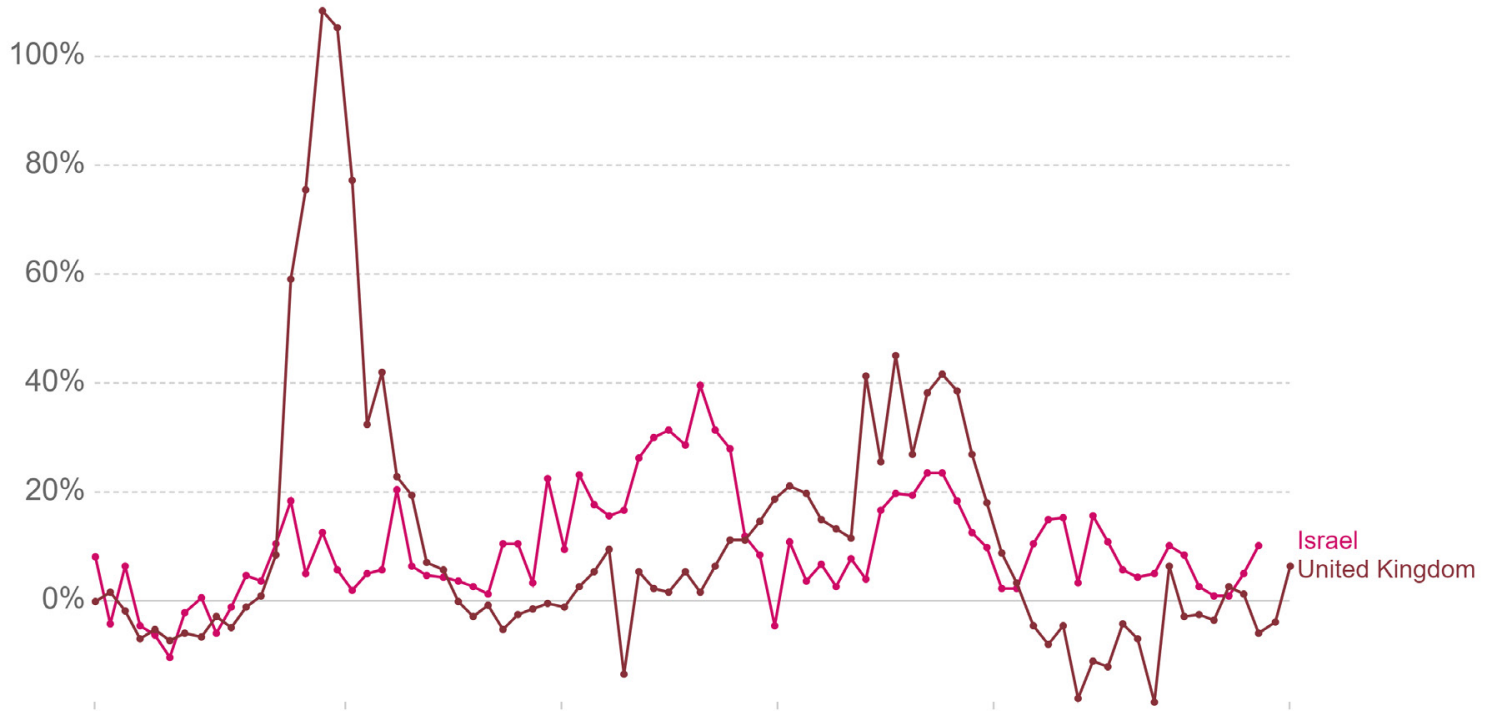

Jan 5,2020

Apr 30, 2020

Aug 8, 2020

Nov 16, 2020

Feb 24, 2021

Jul 11, 2021

Source: Human Mortality Database (2021), World Mortality Dataset (2021) Note: Comparisons across countries are affected by differences in the completeness of death reporting. Details can be found at our Excess Mortality page.

Fig. 2. Excess mortality during COVID-19: deaths from all causes compared with previous years, all ages in the UK and Israel (source: Our World in Data $\left.{ }^{[1]}\right)$.

Note: Limited testing and challenges in the attribution of the cause of death mean that the number of confirmed deaths may not be an accurate count of the true number of deaths from COVID-19. Differences in completeness of investigation of suspected COVID-19 cases, access to healthcare and reporting of deaths prevent head-to-head comparison between some countries.

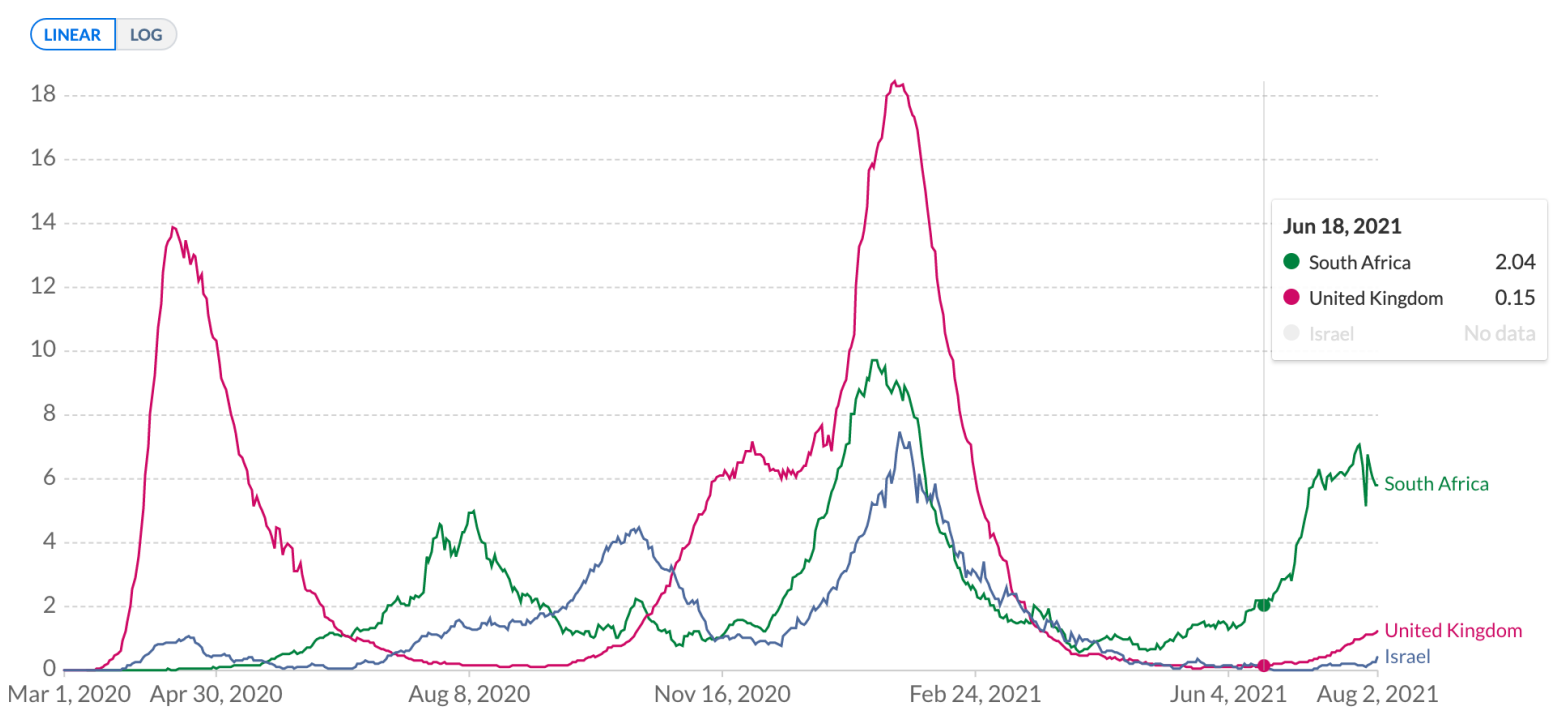

Source: Johns Hopkins University CSSE COVID-19 Data

Fig. 3. Daily new confirmed COVID-19 deaths per million population in South Africa, Israel and the UK (source: Our World in Data ${ }^{[I]}$ ). The rolling 7-day average is shown. 
COVID-19 and death. In contrast, the magnitude of reported deaths in SA during the resurgence being experienced (i.e. third wave) due to the delta variant is likely to be similar to or exceed what was observed during the first or second waves (Fig. 3). This is partly a consequence of SA's having only belatedly started to increase its COVID-19 vaccination rate, with $10.5 \%$ of the population having received at least a single dose by 2 August 2021 .

\section{Conclusions}

In summary, although COVID-19 vaccines confer variable protection against infection and mild COVID-19, they have not interrupted virus transmission, particularly for the highly transmissible delta variant. Based on an $R_{0}$ estimate of 6 for the delta variant, it is unlikely that any country could have a sustainable strategy for durable high level of protection against infection by the delta variant. Mutations of the SARS-CoV-2 genome are likely to continue resulting in enhanced transmissibility, infectiousness and resistance to neutralising activity of antibody induced by the current generation of COVID-19 vaccines.

The decision by the UK government to allow the opening up of society and learning to live with the virus had been made with the comfort of having excelled in ensuring that adults are protected through vaccination against what matters most, i.e. minimising the risk of severe COVID-19 and death, even with ongoing circulation of the virus. The UK approach seemingly concedes that the goal of herd immunity, even in a highly resourced setting, is unattainable. Furthermore, ongoing focus on aspiring to herd immunity by highly resourced countries comes at the cost of further exacerbating inequity that exists in the ability of LMICs to timeously access lifesaving COVID-19 vaccines. Recalibrating our expectations of COVID-19 vaccines is long overdue, and ongoing promotion of booster doses of COVID-19 vaccines where they are least needed in the world, while millions are left susceptible to dying from COVID-19 because they are not able to access vaccines, is immoral.

Consequently, even in the unlikely scenario of vaccinating $84 \%$ of the total SA population, which would require vaccination of $100 \%$ of individuals $>12$ years of age, achieving a herd immunity state with the current generation of COVID-19 vaccines is unlikely. Modelling based on antibody dynamics suggests that booster doses could be required every 2 - 3 years to protect against severe COVID19 , and possibly every 6 - 9 months to protect against any SARSCoV-2 infection. ${ }^{[15]}$ Sustaining repeat booster doses of COVID-19 vaccination for the entire population every $2-3$ years, let alone annually, is not pragmatic. This is particularly so in low-income countries, where just over $1 \%$ of the population has currently received a single dose of the 4 billion doses of COVID-19 vaccines that have been deployed to date, with the global average being $27 \%$ coverage with at least a single dose. ${ }^{[1]}$

Declaration. None.

Acknowledgements. None.

Author contributions. Sole author.

Funding. None.
Conflicts of interest. The author's institution received grants for COVID-19 vaccine and epidemiology studies from the Bill \& Melinda Gates Foundation, the South African Medical Research Council and Novavax.

1. Ritchie H, Ortiz-Ospina E, Beltekian D, et al. Coronavirus pandemic (COVID-19). Our World in Data, 2020. https://ourworldindata.org/covid-cases (accessed 30 July 2021).

2. Sykes W, Mhlanga L, Swanevelder R, et al. Prevalence of anti-SARS-CoV-2 antibodies among blood donors in Northern Cape, KwaZulu-Natal, Eastern Cape, and Free State provinces of South Africa in January 2021. Research Square, 12 February 2021. https://www.researchsquare.com/article/rs-233375/ v1 (accessed 1 August 2021).

3. Anand A, Sandefur J, Subramanian A. Three new estimates of India’s all-cause excess mortality during the COVID-19 pandemic. CGD Working Paper 589. Center for Global Development, 20 July 2021 https://cgdev.org/publication/three-new-estimates-indias-all-cause-excess-mortality-during-covid19-pandemic (accessed 1 August 2021).

4. South African Medical Research Council. Report on weekly deaths in South Africa. www.samrc.ac.za/ reports/report-weekly-deaths-south-africa (accessed 30 July 2021).

5. Yu CJ, Wang ZX, Xu Y, Hu MX, Chen K, Qin G. Assessment of basic reproductive number for COVID-19 at global level: A meta-analysis. Medicine (Baltimore) 2021;100(18):e25837. https://doi. org/10.1097/MD.0000000000025837

6. Farinholt T, Doddapaneni H, Qin X, et al. Transmission event of SARS-CoV-2 Delta variant reveals multiple vaccine breakthrough infections. medRxiv 2021 (epub 12 July 2021). https://doi org $/ 10.1101 / 2021.06 .28 .21258780$

7. Wibmer CK, Ayres F, Hermanus T, et al. SARS-CoV-2 501Y.V2 escapes neutralization by South African COVID-19 donor plasma. Nat Med 2021;27(4):622-625. https://doi.org/10.1038/s41591-021-01285-x Shinde V, Bhikha S, Hoosain Z, et al. Efficacy of NVX-CoV2373 Covid-19 vaccine against the B.1.35 variant. N Engl J Med 2021;384(20):1899-909. https://doi.org/10.1056/NEJMoa2103055

9. Madhi SA, Baillie V, Cutland CL, et al. Efficacy of the ChAdOx1 nCoV-19 Covid-19 vaccine agains the B.1.351 variant. N Engl J Med 2021;384(20):1885-1898. https://doi.org/10.1056/NEJMoa2102214

10. Noori M, Nejadghaderi SA, Arshi S, et al. Potency of BNT162b2 and mRNA-1273 vaccine-induced neutralizing antibodies against severe acute respiratory syndrome-CoV-2 variants of concern A systematic review of in vitro studies. Rev Med Virol 2021:e2277 (epub 19 July 2021). https://doi. org $/ 10.1002 / \mathrm{rmv} .2277$

11. Alter G, Yu J, Liu J, et al. Immunogenicity of Ad26.COV2.S vaccine against SARS-CoV-2 variants in humans. Nature 2021 (epub 9 June 2021). https://doi.org/10.1038/s41586-021-03681-2

12. Earle KA, Ambrosino DM, Fiore-Gartland A, et al. Evidence for antibody as a protective correlate for Earle KA, Ambrosino DM, Fiore-Gartland A, et al. Evidence for antibody as a protective correlate for

COVID-19 vaccines. Vaccine 2021;39(32):4423-4428. https://doi.org/10.1016/j.vaccine.2021.05.063
3. Thomas SJ, Moreira ED, Kitchin N, et al. Six month safety and efficacy of the BNT162b2 mRNA

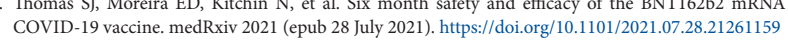
COVID-19 vaccine. medRxiv 2021 (epub 28 July 2021). https://doi.org/10.1101/2021.07.28.21261159
Liu C, Ginn HM, Dejnirattisai W, et al. Reduced neutralization of SARS-CoV-2 B.1.617 by vaccine and 14. Liu C, Ginn HM, Dejnirattisai W, et al. Reduced neutralization of SARS-CoV-2 B.1.617 by vaccine and
convalescent serum. Cell 2021;S0092-8674(21)00755-8 (epub 17 June 2021). https://doi.org/10.1016/j. cell. 2021.06 .020

15. Khoury DS, Cromer D, Reynaldi A, et al. Neutralizing antibody levels are highly predictive of immune protection from symptomatic SARS-CoV-2 infection. Nat Med 2021;27(7):1205-1211. https://do org/10.1038/s41591-021-01377-8

16. Fischer R, van Doremalen N, Adney D, et al. ChAdOx1 nCoV-19 (AZD1222) protects hamsters against SARS-CoV-2 B.1.351 and B.1.1.7 disease. bioRxiv (epub 15 March 2021). https://doi. org/10.1101/2021.03.11.435000

17. Nasreen S, Chung H, He S, et al. Effectiveness of COVID-19 vaccines against variants of concern, Canada. medRxiv 2021 (epub 16 July 2021). https://doi.org/10.1101/2021.06.28.21259420

18. Yao L, Wang GL, Shen Y, et al. Persistence of antibody and cellular immune responses in COVID-19 patients over nine months after infection. J Infect Dis 2021:jiab255 (epub 12 May 2021). https://doi. patients over nine months
org/10.1093/infdis/ijab255

19. Shrotri M, Navaratnam AMD, Nguyen V, et al. Spike-antibody waning after second dose of BNT162b2 or ChAdOx1. Lancet 2021;398(10298):385-387. https://doi.org/10.1016/S0140-6736(21)01642-1

20. Angel Y, Spitzer A, Henig O, et al. Association between vaccination with BNT162b2 and incidence of symptomatic and asymptomatic SARS-CoV-2 infections among health care workers. JAMA 2021;325(24):2457-2465. https://doi.org/10.1001/jama.2021.7152

21. Chodick G, Tene L, Rotem RS, et al. The effectiveness of the TWO-DOSE BNT162b2 vaccine: Analysi of real-world data. Clin Infect Dis 2021:ciab438 (epub 17 May 2021). https://doi.org/10.1093/cid/ ciab438

22. Dagan N, Barda N, Kepten E, et al. BNT162b2 mRNA Covid-19 vaccine in a nationwide mas vaccination setting. N Engl J Med 2021;384(15):1412-1423. https://doi.org/10.1056/NEJMoa2101765

23. Layan M, Gilboa M, Gonen T, et al. Impact of BNT162b2 vaccination and isolation on SARSCoV-2 transmission in Israeli households: An observational study. medRxiv 2021 (epub 16 July 2021). https:// doi.org/10.1101/2021.07.12.21260377

24. Prunas O, Warren JL, Crawford FW, et al. Vaccination with BNT162b2 reduces transmission of SARS-CoV-2 to household contacts in Israel. medRxiv 2021 (epub 16 July 2021). https://doi. org/10.1101/2021.07.13.21260393

25. Levine-Tiefenbrun M, Yelin I, Katz R, et al. Initial report of decreased SARS-CoV-2 viral load after inoculation with the BNT162b2 vaccine. Nat Med 2021;27(5):790-792. https://doi.org/10.1038/ s41591-021-01316-7

26. Lovelace B, jr. Israel says Pfizer Covid vaccine is just $39 \%$ effective as delta spreads, but still prevents severe illness. CNBC, 23 July 2021. https://www.cnbc.com/2021/07/23/delta-variant-pfizer-covidvaccine-39percent-effective-in-israel-prevents-severe-illness.html (accessed 3 August 2021).

Accepted 3 August 2021. 